\title{
Comprehensive literature review of randomized clinical trials examining novel treatment advances in patients with colon cancer
}

\author{
William Paul Skelton IV ${ }^{1} \wedge$, Aaron J. Franke ${ }^{1 \wedge}$, Atif Iqbal ${ }^{2}$, Thomas J. George ${ }^{3 \wedge}$ \\ ${ }^{1}$ Division of Medical Oncology, H. Lee Moffitt Cancer Center and Research Institute, University of South Florida, Florida, USA; ${ }^{2}$ Section of \\ Colorectal Surgery, Baylor College of Medicine, Houston, USA; ${ }^{3}$ Division of Hematology \& Oncology, Department of Medicine, University of \\ Florida College of Medicine, Florida, USA \\ Contributions: (I) Conception and design: All authors; (II) Administrative support: All authors; (III) Provision of study materials or patients: All \\ authors; (IV) Collection and assembly of data: All authors; (V) Data analysis and interpretation: All authors; (VI) Manuscript writing: All authors; (VII) \\ Final approval of manuscript: All authors. \\ Correspondence to: William Paul Skelton IV, MD. H. Lee Moffitt Cancer Center, 12902 Magnolia Drive, Tampa, FL 33602, USA. \\ Email: William.Skelton@moffitt.org; Thomas J. George, MD, FACP. Department of Medicine, Division of Hematology \& Oncology, University of \\ Florida, PO Box 100278, 1600 SW Archer Road Gainesville, FL 32610-0278, USA. Email: Thom.George@medicine.ufl.edu.
}

\begin{abstract}
The treatment of colon cancer has had numerous recent advances, in terms of surgical approach, adjuvant therapies, and more. In this review, the authors examine randomized clinical trials comparing open surgery to laparoscopic surgery (including total mesocolic excision), and also examine the role of robotic surgery. Novel surgical techniques including the no-touch technique, side-to-side anastomosis, suture technique, complete mesocolic excision (CME) with central vascular ligation (CVL), and natural orifice transluminal endoscopic surgery (NOTES) are outlined. The role of placing endoscopic self-expandable metal stents (SEMS) for colonic obstruction is compared and contrasted with the surgical approach, and the effect that the anti-VEGF inhibitor bevacizumab may have on this side effect profile is further explored. The role of the resection of the primary tumor in the setting of metastatic disease is examined with respect to survival benefit. Pathways of perioperative care which can accelerate post-surgical recovery, including enhanced recovery after surgery (ERAS) are examined. The role of adjuvant chemotherapy in patients with high-risk stage II and patients with stage III disease is examined, along with the role on circulating tumor DNA (ctDNA) as well as with the biologic targeted agents cetuximab and bevacizumab. Lastly, the authors detail the postoperative surveillance schedules after surgical resection with respect to survival outcomes.
\end{abstract}

Keywords: Colon cancer; surgery; perioperative care; adjuvant therapy; surveillance

Submitted Apr 11, 2020. Accepted for publication Jul 20, 2020.

doi: 10.21037 /jgo-20-184

View this article at: http://dx.doi.org/10.21037/jgo-20-184

\section{Introduction}

Surgery remains the backbone of curative treatment for colon cancer, but significant advances have been made with adjuvant therapy. Laparoscopic surgery has become increasingly utilized over time. In this review, the authors highlight pivotal studies of historical interest and review contemporary randomized controlled trials (RCTs) that have impacted the clinical management of patients with resectable, non-metastatic colon cancer published since our institution's previous systematic literature review (1).

^ORCID: Dr. William Paul Skelton IV: 0000-0003-4923-8178; Dr. Aaron J. Franke: 0000-0002-7882-9762; Dr. Thomas J. George: 00000002-6249-9180. 


\section{Surgical approach: laparoscopic versus open surgery}

Laparoscopic surgical technique compared with an open approach for colon cancer has been proven to be both technically feasible and has led to similar oncologic outcomes, including lymph node retrieval and surgical margins, highlighted by numerous RCTs, including five level 1a RCTs (2-6). This includes the largest trial on this topic to date (Clinical Outcomes of Surgical TherapyCOST trial) (7). However, the United Kingdom Medical Research Council Conventional versus LaparoscopicAssisted Surgery in Colorectal Cancer (CLASICC) trial noted a statistically insignificant higher rate of positive margins in patients who underwent laparoscopic surgery compared to open surgery. This may be explained by the fact that this trial included patients with rectal cancer in addition to colon cancer, which likely contributed to those results $(4,8)$. Moreover, there were no increased local or distant tumor recurrence rates at three years. No trial other than the initial one reported by Lacy and colleagues (2) had reproduced the short term survival benefit with minimally invasive colectomy.

Additional RCTs and meta-analyses (4-7,9-20) have reinforced and further established benefits and noninferiority of a laparoscopic compared to open approach. A meta-analysis of multiple RCTs and systematic reviews of RCTs found laparoscopic surgery for colon cancer (lap) to be non-inferior to the open approach in terms of overall survival (OS) (HR =1.01, 95\% CI: 0.86-1.19) (20). No significant difference was noted in the number of harvested lymph nodes (range: 5.5-23 lap versus 7.8-26 open). This lack of difference in survival rates was reinforced by the Australasian Laparoscopic Colon Cancer Study Trial, which randomized 601 patients to either laparoscopic-assisted or open approach. The 5-year overall survival (OS) (77.7\% lap vs. $76 \%$ open) and disease-free survival (DFS) (72.7\% lap vs. $71.2 \%$ open) were similar between groups. Patients needing conversion to an open procedure had a higher postoperative infection rate and worse 5-year DFS than either of the groups that were completed laparoscopically or open. This finding mirrors that of the COST trial (7).

The adequacy of 'Total Mesocolic Excision' in the setting of colon cancer, drawing inspiration from the rectal cancer surgical approach in the mesorectum, was assessed in a recent RCT for laparoscopic versus open approach and was found to be similar (9). In fact, a significantly higher proportion of left sided cancer patients operated on laparoscopically had a dissection 'beyond' D3. This has also been found to be the case in the first RCT to compare laparoscopic versus open resection for liver metastases, where a similar rate of resection margins was found, however the laparoscopic group had decreased postoperative complications compared to the open group (21).

Comparing a minimally invasive approach with open surgery, laparoscopy has been found to have numerous short term benefits, including but not limited to minimized use of narcotic pain medications, less post-operative constipation, less EBL (estimated blood loss) periprocedurally, and a shorter hospital stay (2-4,6,22). However, this does not translate into an improvement in long-term quality of life (QOL) $(4,23,24)$ and operative times are longer with laparoscopy (2-4,6,8,22-24). Rates of incisional hernia and adhesion-related small bowel obstruction (SBO) are lower with laparoscopic surgery without an impact on the number of incisional hernia repairs or reoperations for adhesion-related SBO (10). Long term results between the two approaches are similar in terms of OS and DFS (2-7,9-19). Therefore, based on the aforementioned data, in the hands of an experienced surgeon, laparoscopic colectomy for colon cancer can be considered as a safe and effective alternate approach to open surgery.

\section{Robotic versus laparoscopic surgery}

Data comparing robotic and laparoscopic approaches is scant in the surgical literature, most notably one RCT for patients undergoing right colectomy by Park et al. (25). No benefits were found in regards to outcomes or complications. However, the patients in the robotic cohort had higher costs of hospitalization $(\$ 12,235$ vs. $\$ 10,320$, $\mathrm{P}=0.013)$ and longer duration of surgery (195 vs. $130 \mathrm{~min}$, $\mathrm{P}<0.001)$.

In summary, there is currently no RCT evidence to justify a robotic approach for colon cancer over laparoscopic surgery. The role of extraction of a right colon specimen through a pfannenstiel incision (robotic approach) rather than an upper midline (laparoscopic approach), with its potentially lower incisional hernia rates and better pain control should be explored further. It is important to note that although most robotic approaches utilize the pfannenstiel incision, some laparoscopic approaches with intracorporeal anastomosis also do the same. Similarly, recent techniques for transanal extraction of the left sided specimen leading to complete avoidance of an extraction incision, with its inherent advantages, is promising but 
higher levels of evidence are needed in light of the cancer population.

\section{Surgical technique}

Over the past several decades, several aspects of surgical technique have been evaluated in prospective RCTs (26-32) which have helped to define contemporary surgical standards. A RCT failed to demonstrate a significant difference with the no-touch surgical technique when compared with standard practices, though a trend toward improved DFS with the no-touch technique was observed (26). High ligation of the inferior mesenteric artery failed to show a 5 -year survival benefit after segmental and left hemicolectomy compared to routine ligation (27). Handsewn anastomosis compared to stapled anastomosis was associated with an increased rate of radiologically detected leak in a randomized study (28). Sentinel lymph node (SLN) assessment in colon cancer has unreliable results (false negative rates between $7-54 \%$ ) compared to melanoma and breast cancer (33-37), making its reliability and clinical significance of micrometastasis questionable.

Matsuda et al. compared isoperistaltic and aniperistaltic stapled side-to-side anastomosis (SSSA) in a RCT, however this was suspended early after excess morbidity was seen in the isoperistaltic SSSA group (30). No significant differences were noted in the incidence of anastomotic complications or hospital stay between groups but the only two anastomotic leaks were seen in the isoperistaltic group leading to study suspension.

A non-inferiority trial compared subcuticular absorbable suture with interrupted suture for closure of cleancontaminated wounds after resection of the colon cancer (31). All patients received a mechanical and antibiotic bowel preparation. Subcuticular closure was found to be noninferior to interrupted suture closure of the wound $(\mathrm{P}=0.008)$ in terms of incisional surgical-site infection rate $(11 \%$ in both arms).

Many centers have begun to adopt complete mesocolic excision (CME) and central vascular ligation (CVL) in right-sided colon cancers (38). The extended lymph node harvest with CME to more accurately determine stage has shown improved DFS (39). The preferred surgical approach remains a medial to lateral dissection for a more comfortable CVL. There had been some concerns with increased perioperative morbidity and mortality however this was not verified with subsequent studies. In the hands of experienced surgeons, CME/CVL right-sided colectomy with intracorporeal anastomosis may have improved outcomes without a worsening complication rate (40).

Natural orifice transluminal endoscopic surgery (NOTES)-assisted surgery was evaluated in an RCT for laparoscopic left-sided resections with specimen delivered either through the anus using the transanal endoscopic operation (TEO) device versus a mini-laparotomy (32). No significant difference was observed regarding blood loss, time of surgery, or length of hospitalization; however the patients in the NOTES group had less pain one week postoperatively and had no infections $(\mathrm{P}<0.05)$. In summary, these RCTs have supported refinements in surgical practice with a no-touch technique (medial to lateral dissection with early vascular control) and stapled anastomosis currently recommended based on the current available data.

\section{Endoscopic stent versus surgery for colonic obstruction}

Colonic stents [self-expandable metal stents (SEMS)] are used for either palliation of an unresectable/metastatic left sided tumor or to restore luminal patency to prepare patients for elective surgery with primary anastomosis. For the purposes of this review, we will focus on the latter, i.e., the role of stents to avoid surgery in patients with emergent bowel obstructions. Unfortunately, the literature is limited to many non-randomized institutional experiences with without consensus results. We have summarized the results from both the non-randomized and randomized studies, particularly where there are potential conflicting results. Study results are organized in three broad categories: SEMS success, complications, and oncologic results.

In uncontrolled studies, stent placement before elective surgery was suggested to decrease mortality, morbidity, colostomy creation rate and provided the opportunity for neoadjuvant therapy, thus improving patients' prognosis. A systematic review on SEMS revealed an overall success rate of $84-100 \%$ and a success rate of $72 \%$ when utilized as bridge prior to surgery (41). Major stent-related complications were rare (perforation $4 \%$, stent migration $12 \%$, and re-obstruction $7 \%$ ) with a cumulative mortality rate of $0.58 \%$. Discordant oncologic outcomes of stenting versus emergent surgery have shown OS and DFS rates ranging from no difference (41-43), worse outcomes in the stent group $(44,45)$, to few series showing worse outcomes in the emergent surgery group. Such discrepancies supported the need for RCT data assessing the utility of SEMS in this setting. 
The first RCT published on this topic was the Stent-In 2 trial which randomized patients with curable malignant colonic obstruction to emergency surgery or stent placement as a bridge to elective surgery (46). OS and DFS were similar at 4-years but DFS was significantly worse in the patients with stent-related perforation compared to those without a perforation ( $0 \%$ vs. $45 \%, \mathrm{P}=0.007)$. Stent-related perforations were seen in $13 \%$, with occult perforations noted in the resected specimen in an additional $10 \%$ of patients. This first RCT suggested that stent placement was associated with a higher risk of cancer recurrence if a perforation occurred which was also more frequent than previously thought (up to $23 \%$ ), but the events were few.

A meta-analysis of RCTs (47), included as level 1a evidence, had four RCTs of which three (48-50) closed prematurely because of safety and efficacy concerns (one because the emergency surgery group had a significantly increased anastomotic leak rate and two others because of stent-related complications and increased 30-day morbidity following SEMS management). Although stents were associated with a significantly higher successful primary anastomosis rate and lower overall stoma rates, no difference in overall complications (including anastomotic leak and SSI) or mortality was noted in the meta-analysis between groups. Interestingly, the rate of primary anastomosis was similar but the rate of successful primary anastomosis was in favor of SEMS. Similarly, the rates of permanent stoma were similar, but the rate of overall stoma was also in favor of SEMS. No difference in final QOL was noted between groups (one of the above RCTs which was suspended) (49). The low success rates for stenting $(70 \%)$ and high perforation rates (clinical perforation rate: $6.9 \%$, silent perforation rate: $14 \%$ ) raised concern, however cumulative mortality rates after stenting were similar as a bridge to surgery $(6.9 \%)$ versus surgery alone (5.9\%). The authors of the meta-analysis concluded that colonic stenting as a bridge to surgery remained of uncertain clinical significance and should not be routinely used until robust survival data are available, except within the context of a study. Other RCTs published since then have reinforced these results of similar OS and DFS rates between these two groups (48,50-55). These results contradict the findings of early non-randomized studies that showed emergency surgery to be an independent predictor of mortality and morbidity (56). The initial concerns over perforation jeopardizing the margins of a future resection and associated worse survival were not substantiated.
With the anti-VEGF inhibitor bevacizumab being incorporated into more regimens for advanced colon cancer (particularly metastatic disease), it is important to explore how this may affect the side-effect profile of SEMS. A potential of higher risk of perforation was first reported by Cennamo and colleagues in a 2009 case series where they found $2 / 28$ of their patients with SEMS treated with bevacizumab experienced colonic perforation (57). A nine-year longitudinal study at MD Anderson of 199 patients with SEMS found that there was one perforation in the 104 patients $(0.96 \%)$ who had received bevacizumab, compared to 3 perforations in the 95 patients $(3.1 \%)$ who had not received bevacizumab (58). A Spanish retrospective analysis of 78 patients showed that there was a higher complication rate with perforations in patients who had received bevacizumab (2/16, $12.5 \%)$ compared to patients who were treated with chemotherapy alone $(3 / 31,9.7 \%)$ or patients who did not undergo treatment $(2 / 31,6 \%)$, although this was found to not be statistically significant (OR 1.76; 95\% CI: 0.35-8.99, $\mathrm{P}=0.38$ ) (59). It is important to have a risk/benefit discussion with the patient as well as the patient case being discussed in a multi-disciplinary fashion as some patients may be able to live for years. Further large-scale studies are necessary to better delineate the risk of colonic perforation in patients receiving VEGF inhibitors.

In summary, using the aforementioned data, SEMS have been shown to have a greater successful primary anastomotic rate as well as a lower colostomy requirement (albeit short-term), thereby avoiding a second procedure for reversal of the colostomy. Overall, SEMS is an appealing option in spite of the initial concerns over perforation rates, as this has not impacted OS or DFS. A well powered RCT evaluating the pathologic and oncologic outcomes after this approach is needed to validate these recommendations.

\section{Resection of primary tumor in the setting of metastatic disease}

Nationally, the rate of primary tumor resection (PTR) in metastatic colorectal cancer (mCRC) has decreased by $50 \%$ from $1999-2008$, while the use of chemotherapy and multimodality therapy has increased (by $105 \%$ and $27 \%$, respectively) (60). In asymptomatic or minimally symptomatic patients, the role of PTR for avoiding future symptoms or to improve survival had been unclear, compared to the benefits of PTR shown in symptomatic patients with $\mathrm{mCRC}$, which is supported by the current 
guidelines. The concerns over PTR in the metastatic setting are unproven survival benefit, surgery-related morbidity and mortality, possible PTR-related metastatic-tumor growth (based mostly on preclinical data) as well as concern over surgical complications delaying systemic therapy. Proponents of PTR in the metastatic setting highlight the prevention of possible complications related to the primary tumor (such as bleeding, obstruction or perforation), as patients who receive initial systemic therapy without PTR are more likely to develop primary tumor-related complications (61).

There are two ongoing RCTs which are addressing this topic: the CAIRO4 trial and the (UICC stage IV): SYNCHRONOUS trial $(62,63)$. A retrospective analysis of two RCTs that aimed to analyze different chemotherapeutic regimens in $\mathrm{mCRC}$ was done for the prognostic value of PTR in mCRC: CAIRO and CAIRO 2 trials $(64,65)$. Both RCTs showed significantly better OS and progression-free survival (PFS) for the resection group compared to the nonresection group (OS: 18.7 vs. 12.4 months, PFS: 8.6 vs. 6.8 months) (66). This was reiterated in 22 nonrandomized studies, most of which showed improved survival for mCRC patients who underwent PTR (66). However, care is needed in interpreting these results as this was not the primary end point of the RCTs and the reasons for resection and nonresection were not identified. The patients that underwent resection were possibly symptomatic and those that did not undergo resection may be asymptomatic, unresectable or poor operative candidates. These biases could explain the results noted above.

A prospective phase III trial by Kanemitsu and colleagues set to answer whether PTR plus chemotherapy was superior to chemotherapy alone in this population of asymptomatic unresectable mCRC patients. They found a similar OS in the PTR plus chemotherapy arm (25.9 months, $95 \%$ CI: $19.9-31.5)$ to the chemotherapy alone arm (26.7 months, 95\% CI: 21.9-32.5), along with a similar PFS (10.4 months with 95\% CI: 8.6-13.4 in the PTR plus chemotherapy arm, compared to 12.1 months with 95\% CI: 9.4-13.2 in the chemotherapy alone arm) (67).

In summary, the recent JCOG1007 practice changing study by Kanemitsu et al. provide definitive data that PTR followed by chemotherapy does not show a survival benefit compared with chemotherapy alone for asymptomatic patients with mCRC. While we also await the results of the two ongoing RCTs on this subject $(62,63)$, PTR does not appear to have a benefit in this patient population.

\section{Perioperative care/enhanced recovery after surgery (ERAS)}

ERAS or 'fast-track' pathways have been developed to accelerate post-surgical recovery. This multidisciplinary approach includes multiple pathway elements focusing on perioperative interventions including: preoperative education, anesthesia, minimally invasive surgical techniques to reduce stress, early discontinuation of epidural/spinal analgesia, a multimodal analgesic approach with goals to reduce narcotic requirements, balanced fluid therapy, prophylaxis of venous thromboembolism, early oral nutrition, avoidance of nasogastric tube and urinary catheter, prevention of bowel ileus, and early rehabilitation and mobilization utilizing physical therapy if needed (68).

This has been the topic of numerous RCTs and metaanalysis of RCTs (69-84). The two meta-analysis were included as level Ia evidence in our review. Results indicate the efficacy of ERAS compared to conventional postoperative management for elective colon cancer surgery with shorter length of hospitalization and decreased morbidity without increased post-operative complications or readmissions to the hospital $(69,70)$. The significant reduction in the incidence of overall complications is attributable to a decrease in non-surgical complications (cardiovascular, respiratory, urinary tract infections) with implementation of ERAS pathways $(69,70)$. The LAFA trial (laparoscopy in combination with fast track multimodal management) has shown better outcomes in patients with colon cancer when using the ERAS pathway undergoing laparoscopic surgery compared to open (13). Increasingly, laparoscopic surgery is itself being considered an element of the ERAS pathway. A RCT detailed in the previous review showed that the routine use of nasogastric tube drainage was unnecessary (29). Since the last review, two RCTs have evaluated the utility of coffee and gum chewing on postoperative bowel recovery after left sided colorectal cancer resections $(85,86)$. Postoperative gum chewing (three times a day) did not have an impact on bowel recovery but the study was likely underpowered (86). Decaffeinated coffee (three times a day) was associated with significantly quicker return of bowel function with no difference in length of stay compared to the groups that drank coffee with caffeine or water (85).

In summary, utilization of ERAS pathways for colon cancer patients has shown benefit (with a shorter length of hospitalization by 2-3 days as well as decreased morbidity), without harm and in fact, there was not shown 
to be corresponding increase in the rates of readmission. Consideration of nationwide implementation of such pathways will likely improve the quality of care provided.

\section{Adjuvant therapies}

Adjuvant chemotherapy has been shown to have improved survival in colon patients with high-risk stage II and patients with stage III disease. In particular, adjuvant intravenous fluoropyrimidine (FP) for six months duration was well established as a standard. It was shown thereafter that capecitabine has a similar DFS as that achieved with infusional 5-fluorouracil $(5-\mathrm{FU})(\mathrm{P}<0.001)$, as well as that the addition of oxaliplatin to FP therapy (oral or intravenous) is superior to $\mathrm{FP}$ monotherapy with respect to DFS (87-89). Debate continues as to whether adjuvant therapy is beneficial for stage II patients. While several high-risk features are known to confer a worse survival and the benefit of adjuvant therapy remains proportional to the risk of recurrence, RCTs have largely been inadequately powered to detect a survival difference with adjuvant chemotherapy.

However, based on provocative data suggesting that circulating tumor DNA (ctDNA) might serve as a highly objective surrogate for minimum residual disease after surgery (90), the recently activated NRG-GI005 (COBRA) study is testing the role of ctDNA in adjuvant therapy decision making for low-risk stage II colon cancer patients (NCT04068103). The BESPOKE study examined the role of ctDNA in advanced solid tumors (of note, this did not include patients with colon cancer) and found that serial ctDNA levels correlated both with radiological response but also survival (91). A multi-center analysis (CRC-MRD Consortia) investigated ctDNA in 535 patients with early and advanced colorectal cancer, the vast majority (432 patients, $81 \%$ of the cohort) being patients with colon cancer. They found that ctDNA correlated with disease status and also suggested that ctDNA be reliably checked six weeks post-surgery for MRD detection (92). A RCT to determine the incidence of ctDNA in patients with Stage II and III colorectal cancer pre-operatively and to correlate this with outcomes is currently recruiting, the results of which are eagerly anticipated (NCT04050345).

In the last few years, several additional RCTs and pooled analyses have verified the benefit of oxaliplatin as part of a FP-based adjuvant therapy backbone $(93,94)$ and also tested the addition of biologic targeted therapies to adjuvant polychemotherapy, where it is known to be effective in the metastatic setting. These latter studies were critical to conduct given that irinotecan, an effective systemic agent in the metastatic setting, was shown in 3 RCTs to be ineffective or even detrimental when administered in the adjuvant setting (95-97).

Cetuximab (cmab) and bevacizumab (bev) are biologictargeted agents, both of which have improved outcomes when utilized in combination with chemotherapy in mCRC. These agents are monoclonal antibodies targeting the epidermal growth factor receptor (eGFR) (cmab) or the vascular endothelial growth factor (VEGF) (bev), respectively. Two RCTs tested whether the addition of cmab to standard FOLFOX adjuvant chemotherapy for resected stage III colon cancer improved outcomes $(98,99)$. The US NCI-based N0147 study was amended to restrict eligibility to only patients with wild-type K-ras tumors but it terminated early after a second interim analysis demonstrated no benefit when adding cmab (98). Threeyear DFS for patients with wild-type K-ras was $71.5 \%$ with FOLFOX plus cmab and $74.6 \%$ with FOLFOX alone (HR 1.21; 95\% CI: $0.98-1.49 ; \mathrm{P}=0.08)$, suggesting a trend toward harm. There were no subgroups that benefitted from cmab, with increased toxicity and greater detrimental differences in all outcomes in patients older than 70 (Table 1).

The addition of bev to adjuvant polychemotherapy was another clinically relevant and scientifically rational hypothesis to assess (100). The US NCI-based NSABP-C08 study tested the addition of bev to FOLFOX6 in stage II (25\%) and III patients (101). This RCT demonstrated that the addition of bev did not significantly improve 3-year DFS compared to FOLFOX alone $(77.4 \%$ vs. $75.5 \%$; HR 0.89; $95 \%$ CI: 0.76 to $1.04 ; \mathrm{P}=0.15$ ).

For stage III completely resected colon cancer, the contemporary standard of care is 6 months of FP- and oxaliplatin-based adjuvant therapy. However, given the potential permanent neurotoxicity and rare deaths associated with such treatment, a worldwide attempt to reduce the duration of treatment (and associated toxicity) was recently undertaken. The global IDEA Study examined 3 versus 6 months of FOLFOX or CAPOX (capecitabine and oxalipaltin) chemotherapy in a cohort of nearly 13,000 completely resected stage III patients. Results gleaned include that in the low-risk group (60\% of stage III patients), 3 months of CAPOX or FOLFOX is sufficient; while in the high-risk group ( $40 \%$ of stage III patients), CAPOX can be utilized for 3 months, while if FOLFOX is utilized, it should be continued for 6 months (102). These recommendations are based on these chemotherapy 
Table 1 Adjuvant chemotherapy regimens for colon cancer

\begin{tabular}{|c|c|c|}
\hline Name & Regimen and dose & Frequency \\
\hline Roswell Park & LV $500 \mathrm{mg} / \mathrm{m}^{2}$ IV followed by 5 -FU $500 \mathrm{mg} / \mathrm{m}^{2}$ IV weekly $\times 6$ & 8 weeks \\
\hline Capecitabine & $1,250 \mathrm{mg} / \mathrm{m}^{2} \mathrm{PO}$ twice daily $\times 14 \mathrm{~d}$ & 21 days \\
\hline FOLFOX4 & $\begin{array}{l}\text { Oxaliplatin } 85 \mathrm{mg} / \mathrm{m}^{2} \text { IV on day } 1 \text { followed by LV } 200 \mathrm{mg} / \mathrm{m}^{2} / \mathrm{d} \text { IV on days } 1 \text { and } 2 \text { followed by } 5 \text {-FU } \\
400 \mathrm{mg} / \mathrm{m}^{2} / \mathrm{d} \text { IV on days } 1 \text { and } 2 \text { followed by } 5 \text {-FU } 600 \mathrm{mg} / \mathrm{m}^{2} / \mathrm{d} \text { CIVI for } 22 \mathrm{~h} \text { on days } 1 \text { and } 2\end{array}$ & 14 days \\
\hline FLOX & $\begin{array}{l}\text { LV } 500 \mathrm{mg} / \mathrm{m}^{2} \mathrm{IV} \text { followed by } 5 \text {-FU } 500 \mathrm{mg} / \mathrm{m}^{2} \mathrm{IV} \text { on days } 1,8,15,22,29,36 \text { and oxaliplatin } 85 \mathrm{mg} / \mathrm{m}^{2} \mathrm{IV} \text { on } \\
\text { days } 1,15 \text {, and } 29\end{array}$ & 8 weeks \\
\hline CAPOX & Oxaliplatin $100-130 \mathrm{mg} / \mathrm{m}^{2}$ IV on day 1 , capecitabine $1,000 \mathrm{mg} / \mathrm{m}^{2} \mathrm{PO}$ twice daily on days $1-14$ & 21 days \\
\hline
\end{tabular}

LV, leucovorin; IV, intravenous; PO, oral; 5-FU, 5-fluorouracil; CIVI, continuous intravenous infusion.

schedules having decreased toxicities while having a similar 5 -year DFS and OS (103).

\section{Postoperative surveillance schedules}

Multiple RCTs have addressed the question of optimal postoperative surveillance schedule after resection of colon cancer (104-107) including a large RCT by Grossmann and colleagues (108), all of which compared intensive versus less intensive surveillance strategies to determine a survival benefit of early diagnosis of cancer recurrence. No study showed a survival advantage with an intensive surveillance approach compared to the less intensive approach.

Numerous large RCTs have been published on this topic, three of which are of prominence: the FACS trial, CEAWatch trial and GILDA trial (109-112). The large multicenter UK FACS (Follow-up After Colorectal Surgery) trial (110) randomly assigned patients after curative surgery to 1 of 4 groups: carcinoembryonic antigen (CEA) only, CT scan only, CEA + CT scan or minimal follow-up. Intensive imaging or CEA screening each provided an increased rate of curative intent surgical treatment compared with minimal follow-up, that only received testing if symptoms occurred, but no advantage was seen in the CEA and CT scan combination arm. No survival advantage was noted with any of the approaches.

The CEAwatch trial compared usual follow-up care to CEA measurements every two months, with imaging performed if tandem CEA increases were seen (111). The intensive CEA surveillance protocol resulted in the detection of more recurrences and also recurrences that could be treated with curative intent comparted to usual follow-up with the time to detection of recurrent disease being shorter (111). The most recent GILDA trial reiterated findings from multiple previous trials (109). Even though the intensive surveillance schedule was able to diagnose recurrence earlier (significant difference in DFS of 5.9 months favoring intensive surveillance), no benefit in OS or QOL was noted. A randomized phase III PRODIGE 13 trial is ongoing and will compare 5 -year OS after intensive radiological monitoring (abdominal ultrasound, chest/abdomen/pelvis CT, and CEA) with a lower intensity program (abdominal ultrasound and chest $\mathrm{X}$-ray) in patients with resected stage II or III colon or rectal tumors (112).

In summary, the current NCCN surveillance guidelines model the CEA + CT proposed by the FACS trial, although based on the currently published RCTs, while there is improvement in DFS, there has not been shown to be an improvement in OS. We anticipate these recommendations will change in the future with more incorporation of ctDNA becoming more widely adopted.

\section{Conclusions}

For colon cancer, surgery remains the backbone of curative treatment with increasing utilization of laparoscopy. Minimally invasive surgery is associated with many shortterm benefits without worsening oncologic outcomes compared to an open approach. Use of endoscopic stents in the setting of an obstructed cancer continues to be an attractive option due to avoidance of a colostomy and a second procedure despite the risk of perforation, as it does 
not negatively affect OS or DFS. Resection of the primary tumor in patients with stage IV disease should be limited to those with obstructive symptoms for whom a diversion or other less invasive procedure is not feasible. Implementation of ERAS pathways has led to shorter length of hospital stays and decreased morbidity without an increase in readmissions. Adjuvant $\mathrm{FP}$ with the addition of oxaliplatin for 6 months in patients with stage III as well as high-risk stage II colon cancer is associated with improved survival with data suggesting lower risk patients may only need 3 months of poly agent chemotherapy (CAPOX specifically). Oral capecitabine and infusional 5-FU offer a similar DFS in the adjuvant setting. Further RCTs are needed, including those ongoing, to bolster existing evidence as well as provide guidance on currently debated areas.

\section{Acknowledgments}

Funding: None.

\section{Footnote}

Conflict of Interest: All authors have completed the ICMJE uniform disclosure form (available at http://dx.doi. org/10.21037/jgo-20-181). TJG reports Institutional research funding from Merck, Institutional research funding from AstraZeneca/MedImmune, Institutional research funding from Lilly, Institutional research funding from Bayer, during the conduct of the study; Institutional research funding from BMS, Institutional research funding from Incyte, Institutional research funding from Tesaro/ GSK, Institutional research funding from Pharmacyclics, Institutional research funding from Ipsen, Institutional research funding from Seattle Genetics, Institutional research funding from NewLink Genetics, outside the submitted work. The other authors have no conflicts of interest to declare.

Ethical Statement: The authors are accountable for all aspects of the work in ensuring that questions related to the accuracy or integrity of any part of the work are appropriately investigated and resolved.

Open Access Statement: This is an Open Access article distributed in accordance with the Creative Commons Attribution-NonCommercial-NoDerivs 4.0 International License (CC BY-NC-ND 4.0), which permits the non- commercial replication and distribution of the article with the strict proviso that no changes or edits are made and the original work is properly cited (including links to both the formal publication through the relevant DOI and the license). See: https://creativecommons.org/licenses/by-nc-nd/4.0/.

\section{References}

1. Iqbal A, George TJ. Randomized clinical trials in Colon and Rectal Surg Oncol Clin N Am 2017;26:689-704.

2. Lacy AM, Garcia-Valdecasas JC, Delgado S, et al. Laparoscopy-assisted colectomy versus open colectomy for treatment of non-metastatic colon cancer: a randomised trial. Lancet 2002;359:2224-9.

3. Clinical Outcomes of Surgical Therapy Study Group, Nelson H, Sargent DJ, et al. A comparison of laparoscopically assisted and open colectomy for colon cancer. N Engl J Med 2004;350:2050-9.

4. Jayne DG, Guillou PJ, Thorpe H, et al. Randomized trial of laparoscopic-assisted resection of colorectal carcinoma: 3 -year results of the UK MRC CLASICC Trial Group. J Clin Oncol 2007;25:3061-8.

5. Buunen M, Veldkamp R, Hop WC, et al. Survival after laparoscopic surgery versus open surgery for colon cancer: long-term outcome of a randomized clinical trial. Lancet Oncol 2009;10:44-52.

6. Hewett PJ, Allardyce RA, Bagshaw PF, et al. Short-term outcomes of the Australasian randomized clinical study comparing laparoscopic and conventional open surgical treatments for colon cancer: the ALCCaS trial. Ann Surg 2008;248:728-38.

7. Stucky CC, Pockaj BA, Novotny PJ, et al. Long-term follow-up and individual item analysis of quality of life assessments related to laparoscopic-assisted colectomy in the COST trial 93-46-53 (INT 0146). Ann Surg Oncol 2011;18:2422-31.

8. Guillou PJ, Quirke P, Thorpe H, et al. Short-term endpoints of conventional versus laparoscopic-assisted surgery in patients with colorectal cancer (MRC CLASICC trial): multicentre, randomised controlled trial. Lancet 2005;365:1718-26.

9. Nakajima K, Inomata M, Akagi T, et al. Quality control by photo documentation for evaluation of laparoscopic and open colectomy with D3 resection for stage II/III colorectal cancer: Japan Clinical Oncology Group Study JCOG 0404. Jpn J Clin Oncol 2014;44:799-806.

10. Bartels SA, Vlug MS, Hollmann MW, et al. Small bowel obstruction, incisional hernia and survival after 
laparoscopic and open colonic resection (LAFA study). Br J Surg 2014;101:1153-9.

11. Sticca RP, Alberts SR, Mahoney MR, et al. Current use and surgical efficacy of laparoscopic colectomy in colon cancer. J Am Coll Surg 2013;217:56-62.

12. Bagshaw PF, Allardyce RA, Frampton CM, et al. Longterm outcomes of the australasian randomized clinical trial comparing laparoscopic and conventional open surgical treatments for colon cancer: the Australasian Laparoscopic Colon Cancer Study trial. Ann Surg 2012;256:915-9.

13. Vlug MS, Wind J, Hollmann MW, et al. Laparoscopy in combination with fast track multimodal management is the best perioperative strategy in patients undergoing colonic surgery: a randomized clinical trial (LAFA-study). Ann Surg 2011;254:868-75.

14. Jayne DG, Thorpe HC, Copeland J, et al. Five-year follow-up of the Medical Research Council CLASICC trial of laparoscopically assisted versus open surgery for colorectal cancer. Br J Surg 2010;97:1638-45.

15. Braga M, Frasson M, Zuliani W, et al. Randomized clinical trial of laparoscopic versus open left colonic resection. Br J Surg 2010;97:1180-6.

16. Allardyce RA, Bagshaw PF, Frampton CM, et al. Australasian Laparoscopic Colon Cancer Study shows that elderly patients may benefit from lower postoperative complication rates following laparoscopic versus open resection. Br J Surg 2010;97:86-91.

17. Mirza MS, Longman RJ, Farrokhyar F, et al. Longterm outcomes for laparoscopic versus open resection of nonmetastatic colorectal cancer. J Laparoendosc Adv Surg Tech A 2008;18:679-85.

18. Lacy AM, Delgado S, Castells A, et al. The long-term results of a randomized clinical trial of laparoscopyassisted versus open surgery for colon cancer. Ann Surg 2008;248:1-7.

19. Liang JT, Huang KC, Lai HS, et al. Oncologic results of laparoscopic versus conventional open surgery for stage II or III left-sided colon cancers: a randomized controlled trial. Ann Surg Oncol 2007;14:109-17.

20. Martel G, Crawford A, Barkun JS, et al. Expert opinion on laparoscopic surgery for colorectal cancer parallels evidence from a cumulative meta-analysis of randomized controlled trials. PLoS One 2012;7:e35292.

21. Fretland ÅA, Dagenborg VJ, Bjornelv GMW et al. Laparoscopic Versus Open Resection for Colorectal Liver Metastases. Ann Surg 2018;267:199-207.

22. Veldkamp R, Kuhry E, Hop WC, et al. Laparoscopic surgery versus open surgery for colon cancer: short- term outcomes of a randomised trial. Lancet Oncol 2005;6:477-84.

23. Weeks JC, Nelson H, Gelber S, et al. Short-term qualityof-life outcomes following laparoscopic-assisted colectomy vs open colectomy for colon cancer: a randomized trial. JAMA 2002;287:321-8.

24. Janson M, Lindholm E, Anderberg B, et al. Randomized trial of health-related quality of life after open and laparoscopic surgery for colon cancer. Surg Endosc 2007;21:747-53.

25. Park JS, Choi GS, Park SY, et al. Randomized clinical trial of robot-assisted versus standard laparoscopic right colectomy. Br J Surg 2012;99:1219-26.

26. Wiggers T, Jeekel J, Arends JW, et al. No-touch isolation technique in colon cancer: a controlled prospective trial. Br J Surg 1988;75:409-15.

27. Rouffet F, Hay JM, Vacher B, et al. Curative resection for left colonic carcinoma: hemicolectomy vs. segmental colectomy. A prospective, controlled, multicenter trial. French Association for Surgical Research. Dis Colon Rectum 1994;37:651-9.

28. Docherty JG, McGregor JR, Akyol AM, et al. Comparison of manually constructed and stapled anastomoses in colorectal surgery. West of Scotland and Highland Anastomosis Study Group. Ann Surg 1995;221:176-84.

29. Wolff BG, Pembeton JH, van Heerden JA, et al. Elective colon and rectal surgery without nasogastric decompression. A prospective, randomized trial. Ann Surg 1989;209:670-3; discussion: 673-5.

30. Matsuda A, Miyashita M, Matsumoto S, et al. Isoperistaltic versus antiperistaltic stapled side-to-side anastomosis for colon cancer surgery: a randomized controlled trial. J Surg Res 2015;196:107-12.

31. Tanaka A, Sadahiro S, Suzuki T, et al. Randomized controlled trial comparing subcuticular absorbable suture with conventional interrupted suture for wound closure at elective operation of colon cancer. Surgery 2014;155:486-92.

32. Leung AL, Cheung HY, Fok BK, et al. Prospective randomized trial of hybrid NOTES colectomy versus conventional laparoscopic colectomy for left-sided colonic tumors. World J Surg 2013;37:2678-82.

33. Stojadinovic A, Nissan A, Protic M, et al. Prospective randomized study comparing sentinel lymph node evaluation with standard pathologic evaluation for the staging of colon carcinoma: results from the United States Military Cancer Institute Clinical Trials Group Study GI01. Ann Surg 2007;245:846-57. 
34. Bembenek AE, Rosenberg R, Wagler E, et al. Sentinel lymph node biopsy in colon cancer: a prospective multicenter trial. Ann Surg 2007;245:858-63.

35. Kelder W, Braat AE, Karrenbeld A, et al. The sentinel node procedure in colon carcinoma: a multi-centre study in The Netherlands. Int J Colorectal Dis 2007;22:1509-14.

36. Bilchik AJ, DiNome M, Saha S, et al. Prospective multicenter trial of staging adequacy in colon cancer: preliminary results. Arch Surg 2006;141:527-33.

37. Bertagnolli M, Miedema B, Redston M, et al. Sentinel node staging of resectable colon cancer: results of a multicenter study. Ann Surg 2004;240:624-8.

38. Søndenaa K, Qurike P, Hohenberger W, et al. The rationale behind complete mesocolic excision (CME) and a central vascular ligation for colon cancer in open and laparoscopic surgery: proceedings of a consensus conference. Int J Colorectal Dis 2014;29:419-28.

39. Gouvas N, Agalianos C, Papaparaskeva K, et al. Surgery along the embryological planes for colon cancer: a systematic review of complete mesocolic excision. Int J Colorectal Dis 2016;31:1577-94.

40. Olmi S, Oldani A, Cesana G, et al. Surgical Outcomes of Laparoscopic Right Colectomy with Complete Mesocolic Excision. JSLS 2020;24:e2020.00023.

41. Sebastian S, Johnston S, Geoghegan T, et al. Pooled analysis of the efficacy and safety of self-expanding metal stenting in malignant colorectal obstruction. Am J Gastroenterol 2004;99:2051-7.

42. Kavanagh DO, Nolan B, Judge C, etal. A comparative study of short- and medium-term outcomes comparing emergent surgery and stenting as a bridge to surgery in patients with acute malignant colonic obstruction. Dis Colon Rectum 2013;56:433-40.

43. Kim HJ, Huh JW, Kang WS, etal. Oncologic safety of stent as bridge to surgery compared to emergency radical surgery for left-sided colorectal cancer obstruction. Surg Endosc 2013;27:3121-8.

44. Sabbagh C, Browet F, Diouf M, etal. Is stenting as 'a bridge to surgery' an oncologically safe strategy for the management of acute, left-sided, malignant, colonic obstruction? A comparative study with a propensity score analysis. Ann Surg 2013;258:107-15.

45. Gorissen KJ, Tuynman JB, Fryer E, etal. Local recurrence after stenting for obstructing left-sided colonic cancer. Br J Surg 2013;100:1805-9.

46. Sloothaak DA, van den Berg MW, Dijkgraaf MG, et al. Oncological outcome of malignant colonic obstruction in the Dutch Stent-In 2 trial. Br J Surg 2014;101:1751-7.
47. Tan CJ, Dasari BV, Gardiner K. Systematic review and meta-analysis of randomized clinical trials of selfexpanding metallic stents as a bridge to surgery versus emergency surgery for malignant left-sided large bowel obstruction. Br J Surg 2012;99:469-76.

48. Pirlet IA, Slim K, Kwiatkowski F, et al. Emergency preoperative stenting versus surgery for acute left-sided malignant colonic obstruction: a multicenter randomized controlled trial. Surg Endosc 2011;25:1814-21.

49. van Hooft JE, Bemelman WA, Oldenburg B, et al. Colonic stenting versus emergency surgery for acute left-sided malignant colonic obstruction: a multicentre randomised trial. Lancet Oncol 2011;12:344-52.

50. Cheung HY, Chung CC, Tsang WW, et al. Endolaparoscopic approach versus conventional open surgery in the treatment of obstructing left-sided colon cancer: a randomized controlled trial. Arch Surg 2009;144:1127-32.

51. Tung KL, Cheung HY, Ng LW, et al. Endo-laparoscopic approach versus conventional open surgery in the treatment of obstructing left-sided colon cancer: longterm follow-up of a randomized trial. Asian J Endosc Surg 2013;6:78-81.

52. Ghazal AH, El-Shazly WG, Bessa SS, et al. Colonic endolumenal stenting devices and elective surgery versus emergency subtotal/total colectomy in the management of malignant obstructed left colon carcinoma. J Gastrointest Surg 2013;17:1123-9.

53. Fiori E, Lamazza A, Schillaci A, et al. Palliative management for patients with subacute obstruction and stage IV unresectable rectosigmoid cancer: colostomy versus endoscopic stenting: final results of a prospective randomized trial. Am J Surg 2012;204:321-6.

54. Ho KS, Quah HM, Lim JF, et al. Endoscopic stenting and elective surgery versus emergency surgery for left-sided malignant colonic obstruction: a prospective randomized trial. Int J Colorectal Dis 2012;27:355-62.

55. Alcántara M, Serra-Aracil X, Falcó J, et al. Prospective, controlled, randomized study of intraoperative colonic lavage versus stent placement in obstructive left-sided colonic cancer. World J Surg 2011;35:1904-10.

56. Morris EJA, Taylor EF, Thomas JD, et al. Thirty-day postoperative mortality after colorectal cancer surgery in England. Gut 2011;60:806-13.

57. Cennamo V, Fuccio L, Mutri V, et al. Does stent placement for advanced colon cancer increase the risk of perforation during bevacizumab-based therapy. Clin Gastroenterol Hepatol 2009;7:1174-6. 
58. Lee JH, Emelogu I, Kukreja K, et al. Safety and efficacy of metal stents for malignant colonic obstruction in patients treated with bevacizumab. Gastrointest Endosc 2019;90:116-24.

59. Pacheco-Barcia V, Mondejar R, Martinez-Saez O, et al. Safety and Oncologic Outcomes of Bevacizumab Therapy in Patients With Advanced Colorectal Cancer and Self-expandable Metal Stents. Clin Colorectal Cancer 2019;18:e287-93.

60. Massarweh NN, Li LT, Sansgiry S, et al. Primary Tumor Resection and Multimodality Treatment for Patients with Metastatic Colon Cancer. Ann Surg Oncol 2016;23:1815-23.

61. Stillwell AP, Buettner PG, Ho YH. Meta-analysis of survival of patients with stage IV colorectal cancer managed with surgical resection versus chemotherapy alone. World J Surg 2010;34:797-807.

62. Rahbari NN, Lordick F, Fink C, et al. Resection of the primary tumour versus no resection prior to systemic therapy in patients with colon cancer and synchronous unresectable metastases (UICC stage IV): SYNCHRONOUS - a randomised controlled multicentre trial (ISRCTN30964555). BMC Cancer 2012;12:142.

63. 't Lam-Boer J, Mol L, Verhoef C, et al. The CAIRO4 study: the role of surgery of the primary tumour with few or absent symptoms in patients with synchronous unresectable metastases of colorectal cancer: a randomized phase III study of the Dutch Colorectal Cancer Group (DCCG). BMC Cancer 2014;14:741.

64. Koopman M, Antonini NF, Douma J, et al. Sequential versus combination chemotherapy with capecitabine, irinotecan, and oxaliplatin in advanced colorectal cancer (CAIRO): a phase III randomised controlled trial. Lancet 2007;370:135-42.

65. Tol J, Koopman M, Rodenburg CJ, et al. A randomised phase III study on capecitabine, oxaliplatin and bevacizumab with or without cetuximab in first-line advanced colorectal cancer, the CAIRO2 study of the Dutch Colorectal Cancer Group (DCCG). An interim analysis of toxicity. Ann Oncol 2008;19:734-8.

66. Venderbosch S, de Wilt JH, Teerenstra S, et al. Prognostic value of resection of primary tumor in patients with stage IV colorectal cancer: retrospective analysis of two randomized studies and a review of the literature. Ann Surg Oncol 2011;18:3252-60.

67. Kanemitsu Y, Shitara K, Mizusawa, et al. A randomized phase III trial comparing primary tumor resection plus chemotherapy with chemotherapy alone in incurable stage IV colorectal cancer: JCOG1007 study (iPACS). 2020 Gastrointestinal Cancers Symposium. J Clin Oncol 2020;38:abstr 7.

68. Feldman LS, Baldini G, Lee L, et al. Enhanced recovery pathways: organization of evidence-based, fast-track perioperative care. Sci Am Surg 2013;1-29.

69. Greco M, Capretti G, Beretta L, et al. Enhanced recovery program in colorectal surgery: a metaanalysis of randomized controlled trials. World J Surg 2014;38:1531-41.

70. Spanjersberg WR, Reurings J, Keus F, et al. Fast track surgery versus conventional recovery strategies for colorectal surgery. Cochrane Database Syst Rev 2011;(2):CD007635.

71. Ren L, Zhu D, Wei Y, et al. Enhanced Recovery After Surgery (ERAS) program attenuates stress and accelerates recovery in patients after radical resection for colorectal cancer: a prospective randomized controlled trial. World J Surg 2012;36:407-14.

72. Wang G, Jiang ZW, Xu J, et al. Fast-track rehabilitation program vs conventional care after colorectal resection: a randomized clinical trial. World J Gastroenterol 2011;17:671-6.

73. Wang Q, Suo J, Jiang J, et al. Effectiveness of fast track rehabilitation vs conventional care in laparoscopic colorectal resection for elderly patients: a randomized trial. Colorectal Dis 2012;14:1009-13.

74. Anderson $\mathrm{AD}, \mathrm{McNaught} \mathrm{CE}, \mathrm{MacFie} \mathrm{J}$, et al. Randomized clinical trial of multimodal optimization and standard perioperative surgical care. Br J Surg 2003;90:1497-504.

75. Delaney CP, Zutshi M, Senagore AJ, et al. Prospective, randomized, controlled trial between a pathway of controlled rehabilitation with early ambulation and diet and traditional postoperative care after laparotomy and intestinal resection. Dis Colon Rectum 2003;46:851-9.

76. García-Botello S, Cánovas de Lucas R, Tornero C, et al. Implementation of a perioperative multimodal rehabilitation protocol in elective colorectal surgery. A prospective randomised controlled study. Cir Esp 2011;89:159-66.

77. Gatt M, Anderson ADG, Reddy BS, et al. Randomized clinical trial of multimodal optimization of surgical care in patients undergoing major colonic resection. Br J Surg 2005;92:1354-62.

78. Ionescu D, Iancu C, Ion D, et al. Implementing fast-track protocol for colorectal surgery: a prospective randomized clinical trial. World J Surg 2009;33:2433-8. 
79. Khoo CK, Vickery CJ, Forsyth N, et al. A prospective randomized controlled trial of multimodal perioperative management protocol in patients undergoing elective colorectal resection for cancer. Ann Surg 2007;245:867-72.

80. Lee TG, Kang SB, Kim DW, et al. Comparison of early mobilization and diet rehabilitation program with conventional care after laparoscopic colon surgery: a prospective randomized controlled trial. Dis Colon Rectum 2011;54:21-8.

81. Muller S, Zalunardo MP, Hubner M, et al. A fast-track program reduces complications and length of hospital stay after open colonic surgery. Gastroenterology 2009;136:842-7.

82. Serclová Z, Dytrych P, Marvan J, et al. Fast-track in open intestinal surgery: prospective randomized study (Clinical Trials Gov Identifier no. NCT00123456). Clin Nutr 2009;28:618-24.

83. Yang $\mathrm{D}, \mathrm{He} \mathrm{W}$, Zhang $\mathrm{S}$, et al. Fast-track surgery improves postoperative clinical recovery and immunity after elective surgery for colorectal carcinoma: randomized controlled clinical trial. World J Surg 2012;36:1874-80.

84. Wang G, Jiang Z, Zhao K, et al. Immunologic response after laparoscopic colon cancer operation within an enhanced recovery program. J Gastrointest Surg 2012;16:1379-88.

85. Dulskas A, Klimovskij M, Vitkauskiene M, et al. Effect of Coffee on the Length of Postoperative Ileus After Elective Laparoscopic Left-Sided Colectomy: A Randomized, Prospective Single-Center Study. Dis Colon Rectum 2015;58:1064-9.

86. Kobayashi T, Masaki T, Kogawa K, et al. Efficacy of Gum Chewing on Bowel Movement After Open Colectomy for Left-Sided Colorectal Cancer: A Randomized Clinical Trial. Dis Colon Rectum 2015;58:1058-63.

87. Twelves C, Wong A, Nowacki MP, et al. Capecitabine as adjuvant treatment for stage III colon cancer. $\mathrm{N}$ Engl J Med 2005;352:2696-704.

88. André T, Boni C, Mounedji-Boudiaf L, et al. Oxaliplatin, fluorouracil, and leucovorin as adjuvant treatment for colon cancer. N Engl J Med 2004;350:2343-51.

89. Kuebler JP, Wieand HS, O'Connell MJ, et al. Oxaliplatin combined with weekly bolus fluorouracil and leucovorin as surgical adjuvant chemotherapy for stage II and III colon cancer: results from NSABP C-07. J Clin Oncol 2007;25:2198-204.

90. Tie J, Wang Y, Tomasetti C, et al. Circulating tumor DNA analysis detects minimal residual disease and predicts recurrence in patients with stage II colon cancer. Sci Transl
Med 2016;8:346ra92.

91. Iafolla MA, Yang C, Dashner S, et al. Bespoke Circulating Tumor DNA (ctDNA) Analysis as a Predictive Biomarker in Solid Tumor Patients (pts) Treated with Single Agent Pembrolizumab. Am Soc Clin Oncol 2019; abstract 2542.

92. Kasi PM, Dayyani F, Morris V, et al. Tumor-Informed Assessment of Molecular Residual Disease and its Incorporation into Practice for Patients with Early and Advanced Stage Colorectal Cancer (CRC-MRD Consortia). Am Soc Clin Oncol 2020;poster 100.

93. Schmoll HJ, Tabernero J, Maroun J, et al. Capecitabine Plus Oxaliplatin Compared With Fluorouracil/Folinic Acid As Adjuvant Therapy for Stage III Colon Cancer: Final Results of the NO16968 Randomized Controlled Phase III Trial. J Clin Oncol 2015;33:3733-40.

94. Schmoll HJ, Twelves C, Sun W, et al. Effect of adjuvant capecitabine or fluorouracil, with or without oxaliplatin, on survival outcomes in stage III colon cancer and the effect of oxaliplatin on post-relapse survival: a pooled analysis of individual patient data from four randomised controlled trials. Lancet Oncol 2014;15:1481-92.

95. Saltz LB, Niedzwiecki D, Hollis D, et al. Irinotecan fluorouracil plus leucovorin is not superior to fluorouracil plus leucovorin alone as adjuvant treatment for stage III colon cancer: results of CALGB 89803. J Clin Oncol 2007;25:3456-61.

96. Van Cutsem E, Labianca R, Bodoky G, et al. Randomized phase III trial comparing biweekly infusional fluorouracil/ leucovorin alone or with irinotecan in the adjuvant treatment of stage III colon cancer: PETACC-3. J Clin Oncol 2009;27:3117-25.

97. Ychou M RJ, Douillard JY, et al. A phase III randomized trial of LV5FU2+CPT-11 vs LV5FU2 alone in adjuvant high risk colon cancer (FNCLCC Accord02/FFCD9802). J Clin Oncol 2005;23:3502a.

98. Alberts SR, Sargent DJ, Nair S, et al. Effect of oxaliplatin, fluorouracil, and leucovorin with or without cetuximab on survival among patients with resected stage III colon cancer: a randomized trial. JAMA 2012;307:1383-93.

99. Taieb J, Tabernero J, Mini E, et al. Oxaliplatin, fluorouracil, and leucovorin with or without cetuximab in patients with resected stage III colon cancer (PETACC-8): an open-label, randomised phase 3 trial. Lancet Oncol 2014;15:862-73.

100.de Gramont A, Van Cutsem E, Schmoll HJ, et al. Bevacizumab plus oxaliplatin-based chemotherapy as adjuvant treatment for colon cancer (AVANT): a phase 3 randomised controlled trial. Lancet Oncol 
2012;13:1225-33.

101.Allegra CJ, Yothers G, O’Connell MJ, et al. Phase III trial assessing bevacizumab in stages II and III carcinoma of the colon: results of NSABP protocol C-08. J Clin Oncol 2011;29:11-6.

102. André T, Iveson T, Labianca R, et al. The IDEA (International Duration Evaluation of Adjuvant Chemotherapy) Collaboration: Prospective Combined Analysis of Phase III Trials Investigating Duration of Adjuvant Therapy with the FOLFOX (FOLFOX4 or Modified FOLFOX6) or XELOX (3 versus 6 months) Regimen for Patients with Stage III Colon Cancer: Trial Design and Current Status. Curr Colorectal Cancer Rep 2013;9:261-9.

103. Sobrero A, Andre T, Meyerhardt J, et al. OS and Longterm DFS of 3 versus 6 months of adjuvant oxaliplatin and fluoropyrimidine-based therapy for patients with Stage III colon cancer: final results from the IDEA collaboration. ASCO Annual Meeting 2020:abstract 4004.

104. Kjeldsen BJ, Kronborg O, Fenger C, et al. A prospective randomized study of follow-up after radical surgery for colorectal cancer. Br J Surg 1997;84:666-9.

105. Mäkelä JT, Laitinen SO, Kairaluoma MI. Five-year followup after radical surgery for colorectal cancer. Results of a prospective randomized trial. Arch Surg 1995;130:1062-7. 106. Ohlsson B, Breland U, Ekberg H, et al. Follow-up after curative surgery for colorectal carcinoma. Randomized

Cite this article as: Skelton WP 4th, Franke AJ, Iqbal A, George TJ. Comprehensive literature review of randomized clinical trials examining novel treatment advances in patients with colon cancer. J Gastrointest Oncol 2020;11(4):790-802 . doi: 10.21037/jgo-20-184 comparison with no follow-up. Dis Colon Rectum 1995;38:619-26.

107. Schoemaker D, Black R, Giles L, et al. Yearly colonoscopy, liver CT, and chest radiography do not influence 5-year survival of colorectal cancer patients. Gastroenterology 1998;114:7-14.

108. Grossmann EM, Johnson FE, Virgo KS, et al. Follow-up of colorectal cancer patients after resection with curative intent-the GILDA trial. Surg Oncol 2004;13:119-24.

109. Rosati G, Ambrosini G, Barni S, et al. A randomized trial of intensive versus minimal surveillance of patients with resected Dukes B2-C colorectal carcinoma. Ann Oncol 2016;27:274-80.

110. Primrose JN, Perera R, Gray A, et al. Effect of 3 to 5 years of scheduled CEA and CT follow-up to detect recurrence of colorectal cancer: the FACS randomized clinical trial. JAMA 2014;311:263-70.

111. Verberne CJ, Zhan Z, van den Heuvel E, et al. Intensified follow-up in colorectal cancer patients using frequent Carcino-Embryonic Antigen (CEA) measurements and CEA-triggered imaging: Results of the randomized CEAwatch" trial. Eur J Surg Oncol 2015;41:1188-96.

112.Lepage C, Phelip JM, Cany L, et al. Effect of 5 years of imaging and CEA follow-up to detect recurrence of colorectal cancer: The FFCD PRODIGE 13 randomised phase III trial. Dig Liver Dis 2015;47:529-31. 